2

3

\title{
Differential expression of TNS1 in the brain metastases of patients with breast cancer.
}

Shahan Mamoor, MS1

${ }^{1}$ shahanmamoor@gmail.com

East Islip, NY USA

Metastasis to the brain is a complication of breast cancer ${ }^{1,2}$ with limited treatment options 3 . We mined published microarray data 4,5 to discover genes associated with brain metastases in patients with metastatic breast cancer. We identified significant differential expression of the gene encoding tensin 1, TNS1, in the brain metastases of patients with breast cancer as compared to primary tumors of the breast. TNS1 may be relevant to the underlying biology by which tumor cells of the breast spread to the brain.

Keywords: TNS1, breast cancer, brain metastases, central nervous system metastases, systems biology of breast cancer, targeted therapeutics in breast cancer. 
One study documented a $34 \%$ incidence of metastases to the central nervous system in breast cancer patients treated with trastuzumab ${ }^{6}$. An unbiased and systems-level understanding of the transcriptional makeup of brain metastatic tissues can support identification of therapeutic targets. We performed global transcriptional profiling of brain metastases in patients with brain metastatic breast cancer6-8. We discovered significant differential expression of TNS1 in brain metastases from patients with brain metastatic breast cancer.

\section{Methods}

We used datasets GSE1259894 and GSE438375 for this global differential gene expression analysis of brain metastatic breast cancer in conjunction with GEO2R. GSE125989 was generated using Affymetrix Human Genome U133A 2.0 Array technology with $n=16$ primary tumors from patients with breast cancer and $n=16$ brain metastases from patients with brain metastatic breast cancer. GSE43837 was generated using Affymetrix Human X3P Array technology with $n=19$ primary breast tumors and $n=19$ brain metastases from patients with breast cancer, analysis performed using platform GPL1352. The Benjamini and Hochberg method of $p$-value adjustment was used for ranking of differential expression but raw $p$-values were used to assess statistical significance of global differential expression. Log-transformation of data was auto-detected, and the NCBI generated category of platform annotation was used. A statistical test was performed to evaluate whether TNS1 gene expression was significantly between primary breast tumors and brain metastases in humans with breast cancer using a two-tailed, unpaired t-test with Welch's correction. We used PRISM for all statistical analyses of differential gene expression in human breast cancer (Version 8.4.0)(455).

\section{Results}

We sought to discover genes associated with metastasis to the brain in patients with breast cancer by mining published microarray datasets 4,5 , comparing global gene expression profiles of primary tumors of the breast to brain metastatic tissues from patients with breast cancer.

\section{TNS1 is differentially expressed in the brain metastases of patients with brain metastatic breast cancer.}

We identified the gene encoding tensin 1, TNS1, as among the genes whose expression was most quantitatively different in brain metastases when compared to primary tumors of the breast in patients with breast cancer ${ }^{4}$. When sorting each of the genes expressed in brain metastases based on significance of difference in expression as compared to primary tumors of the breast, TNS1 ranked 59 out of 22277 total transcripts (Table 1), equating to $99.7 \%$ differential expression. Differential expression of TNS1 in brain metastases in patients with breast cancer was statistically significant (Table $1 ; p=1.16 \mathrm{E}-04$ ).

We queried a second microarray dataset ${ }^{5}$ to attempt to validate transcriptome-wide differential expression of TNS1 in brain metastatic breast cancer. We again found that TNS1 was among the genes whose expression was most quantitatively different when comparing primary tumors of the breast to brain metastases from patients with metastatic breast cancer. When sorting each of the genes expressed in brain metastases based on significance of difference in expression as compared to primary tumors of the breast, TNS1 ranked 369 out of 61359 total transcripts (Table 2), equating to $99.4 \%$ differential expression. Differential expression of TNS1 in brain metastases in patients with breast cancer was statistically significant (Table $2 ; \mathrm{p}=0.00192442$ ). 
TNS1 is expressed at significantly lower levels in the brain metastases of patients with brain metastatic breast cancer as compared to primary tumors of the breast.

We obtained exact mRNA expression levels for TNS1 in primary tumors of the breast and in brain metastases to evaluate magnitude and direction of change in TNS1 gene expression in brain metastatic tissues. TNS1 was expressed at lower levels in brain metastases from patients with breast cancer as compared to primary tumors of the breast (Figure 1). Decreased expression of TNS1 in brain metastases as compared to primary tumors of the breast was statistically significant (Figure 1: $p=0.0003$ ). We calculated a mean fold change of $0.800 \pm 0.142$ in TNS1 expression in brain metastases when compared to primary tumors of the breast (Table 1).

Thus, through comparative transcriptome analysis of tumor and metastatic tumor tissues of patients with breast cancer using published microarray data ${ }^{4,5}$, we found that TNS1 was among the genes most differentially expressed in brain metastases, with significantly decreased expression in brain metastatic tissues as compared to primary tumors of the breast.

\section{Discussion}

To facilitate discovery of novel therapeutic targets in brain metastatic breast cancer, and to more throughly understand the biology of metastasis to the brain in breast cancer, we mined published microarray data 4,5 to identify genes most differentially expressed in the brain metastases of patients with metastatic breast cancer.

Tensin 1, or tensin, is a cytoskeletal component of focal adhesions and actin-binding protein containing a Src homology 2 (SH2) domain 7 . Tensin can interact with filamentous actin (F-actin), and impair the rate at which F-actin polymerizes in actin filaments ${ }^{8}$ by binding to the barbed-end of the actin filament ${ }^{9}$. Tensin associates with focal adhesions through PTB motif-binding to the tail of integrin $\beta 1$ at NPxY motifs ${ }^{10}$. The AMP-activated protein kinase (AMPK) is a negative regulator of tensin expression ${ }^{11}$. Tensin can co-localize with the p130Cas protein at focal adhesions at the leading-edge of migrating cells ${ }^{12}$. Thus, it appears that down-regulation of tensin expression (rather than up-regulation) is important for brain metastatic tissues. This may be to facilitate colonization of the central nervous system by influencing focal adhesion interactions with integrins, or it may be that down-regulation of tensin enables a higher rate of $\mathrm{F}$-actin polymerization during migratory behaviors.

We found here using published microarray data ${ }^{4,5}$ that TNS1 is among the genes whose expression changes most significantly in the brain metastases of patients with metastatic breast cancer and present at significantly lower quantities at the mRNA level in brain metastatic tissue. 


\section{References}

1. Lin, N.U., Amiri-Kordestani, L., Palmieri, D., Liewehr, D.J. and Steeg, P.S., 2013. CNS metastases in breast cancer: old challenge, new frontiers.

2. Tsukada, Y., Fouad, A., Pickren, J.W. and Lane, W.W., 1983. Central nervous system metastasis from breast carcinoma autopsy study. Cancer, 52(12), pp.2349-2354.

3. Robson, M., Im, S.A., Senkus, E., Xu, B., Domchek, S.M., Masuda, N., Delaloge, S., Li, W., Tung, N., Armstrong, A. and Wu, W., 2017. Olaparib for metastatic breast cancer in patients with a germline BRCA mutation. New England Journal of Medicine, 377(6), pp.523-533.

4. Iwamoto, T., Niikura, N., Ogiya, R., Yasojima, H., Watanabe, K.I., Kanbayashi, C., Tsuneizumi, M., Matsui, A., Fujisawa, T., Iwasa, T. and Shien, T., 2019. Distinct gene expression profiles between primary breast cancers and brain metastases from pair-matched samples. Scientific reports, $9(1)$, pp. $1-8$.

5. McMullin, R.P., Wittner, B.S., Yang, C., Denton-Schneider, B.R., Hicks, D., Singavarapu, R., Moulis, S., Lee, J., Akbari, M.R., Narod, S.A. and Aldape, K.D., 2014. A BRCA1 deficient-like signature is enriched in breast cancer brain metastases and predicts DNA damage-induced poly (ADP-ribose) polymerase inhibitor sensitivity. Breast Cancer Research, 16(2), pp.1-10.

6. Bendell, J.C., Domchek, S.M., Burstein, H.J., Harris, L., Younger, J., Kuter, I., Bunnell, C., Rue, M., Gelman, R. and Winer, E., 2003. Central nervous system metastases in women who receive trastuzumab-based therapy for metastatic breast carcinoma. Cancer, 97(12), pp.2972-2977.

7. Davis, S., Lu, M.L., Lo, S.H., Lin, S., Butler, J.A., Druker, B.J., Roberts, T.M., An, Q. and Chen, L.B., 1991. Presence of an SH2 domain in the actin-binding protein tensin. Science, 252(5006), pp. $712-715$.

8. CHEN, H., ISHII, A., WONG, W.K., CHEN, L.B. and Lo, S.H., 2000. Molecular characterization of human tensin. Biochemical Journal, 351(2), pp.403-411.

9. Lo, S.H., Janmey, P.A., Hartwig, J.H. and Chen, L.B., 1994. Interactions of tensin with actin and identification of its three distinct actin-binding domains. The Journal of cell biology, 125(5), pp. 1067-1075.

10. McCleverty, C.J., Lin, D.C. and Liddington, R.C., 2007. Structure of the PTB domain of tensin1 and a model for its recruitment to fibrillar adhesions. Protein science, 16(6), pp.1223-1229.

PAGE 4 
11. Georgiadou, M., Lilja, J., Jacquemet, G., Guzmán, C., Rafaeva, M., Alibert, C., Yan, Y., Sahgal, P., Lerche, M., Manneville, J.B. and Mäkelä, T.P., 2017. AMPK negatively regulates tensin-dependent integrin activity. Journal of Cell Biology, 216(4), pp.1107-1121.

12. Zhao, Z., Tan, S.H., Machiyama, H., Kawauchi, K., Araki, K., Hirata, H. and Sawada, Y., 2016. Association between tensin 1 and p130Cas at focal adhesions links actin inward flux to cell migration. Biology open, 5(4), pp.499-506.

PAGE 5 
Table 1: Differential expression of TNS1 in brain metastases in patients with metastatic breast cancer.

Rank of differential expression, probe ID, $p$-value with respect to differential expression, $t$, a moderated tstatistic, B, the log-odds of differential expression between the two groups compared, fold change (FC) of TNS1 expression between the brain metastases of patients with breast cancer and primary tumors of the breast, gene and gene name are listed in this chart.

PAGE 6 
Rank of differential expression, probe ID, $p$-value with respect to differential expression, $t$, a moderated tstatistic, B, the log-odds of differential expression between the two groups compared, gene and gene name are listed in this chart.

\begin{tabular}{|c|c|c|c|c|c|c|}
\hline Rank & ID & $p$-value & $\mathbf{t}$ & B & Gene & Gene name \\
\hline 369 & Hs.9973.1.A1_3p_at & 0.00192442 & -3.305227 & -1.35773 & TNS1 & tensin 1 \\
\hline
\end{tabular}

Table 2: Differential expression of TNS1 in brain metastases in patients with metastatic breast cancer. 


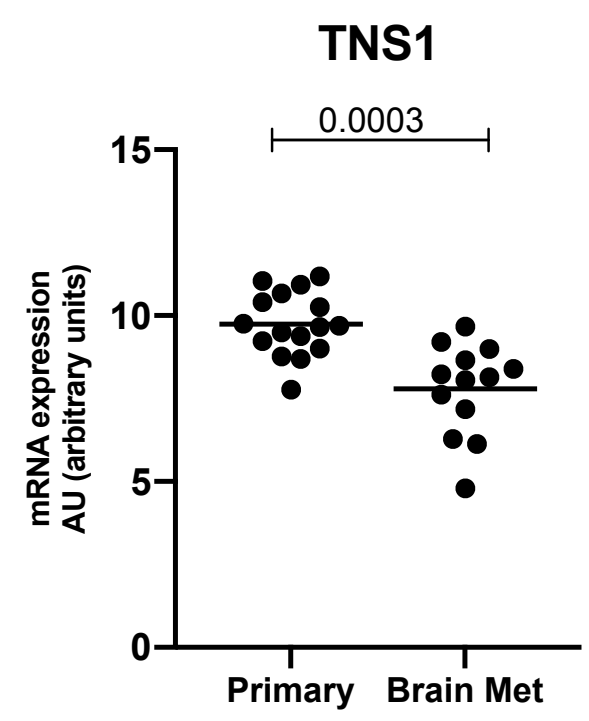

Figure 1: TNS1 is expressed at significantly lower levels in brain metastases as compared to primary tumors of the breast in patients with brain metastatic breast cancer.

The mRNA expression level of TNS1 in primary tumors of the breast (left) and in the brain metastases of brain metastatic breast cancer patients (right) is graphically represented here with mean TNS1 levels marked and the result of a statistical test evaluating difference in mRNA expression level between brain metastases and primary tumors of the breast, a $p$-value, listed above. 